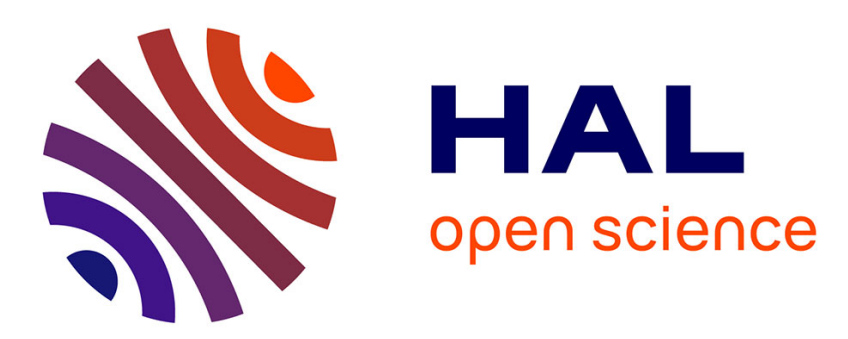

\title{
Wall-Resolved Large Eddy Simulation of a flow through a square-edged orifice in a round pipe at $\mathrm{Re}=\mathbf{2 5 0 0 0}$
}

\author{
S. Benhamadouche, M. Arenas, W.J. J Malouf
}

\section{To cite this version:}

S. Benhamadouche, M. Arenas, W.J. J Malouf. Wall-Resolved Large Eddy Simulation of a flow through a square-edged orifice in a round pipe at $R e=25000$. Nuclear Engineering and Design, 2017, 312, pp.128 - 136. 10.1016/j.nucengdes.2016.09.010 . hal-01715658

\section{HAL Id: hal-01715658 \\ https://hal.science/hal-01715658}

Submitted on 22 Feb 2018

HAL is a multi-disciplinary open access archive for the deposit and dissemination of scientific research documents, whether they are published or not. The documents may come from teaching and research institutions in France or abroad, or from public or private research centers.
L'archive ouverte pluridisciplinaire HAL, est destinée au dépôt et à la diffusion de documents scientifiques de niveau recherche, publiés ou non, émanant des établissements d'enseignement et de recherche français ou étrangers, des laboratoires publics ou privés. 


\title{
Wall-Resolved Large Eddy Simulation of a flow through a square-edged orifice in a round pipe at $\mathrm{Re}=\mathbf{2 5 0 0 0}$
}

\author{
S. Benhamadouche ${ }^{\mathrm{a}, *}$, M. Arenas ${ }^{\mathrm{a}}$, W.J. Malouf ${ }^{\mathrm{a}}$ \\ ${ }^{a}$ Electricité de France, R\&D Division, 6, Quai Watier, 78401 Chatou, France \\ * Corresponding author. Email address: sofiane.benhamadouche@edf.fr
}

\begin{abstract}
The orifice plate is a pressure differential device frequently used for flow measurements in pipes across different industries. The present study demonstrates the accuracy obtainable using a wallresolved Large Eddy Simulation (LES) approach to predict the velocity, the Reynolds stresses, the pressure loss and the discharge coefficient for a flow through a square-edged orifice in a round pipe at a Reynolds number of 25000 . The ratio of the orifice diameter to the pipe diameter is $\beta=$ 0.62 , and the ratio of the orifice thickness to the pipe diameter is 0.11 . The mesh is sized using refinement criteria at the wall and preliminary RANS results to ensure that the solution is resolved beyond an estimated Taylor micro-scale. The inlet condition is simulated using a recycling method, and the LES is run with a dynamic Smagorinsky sub-grid scale (SGS) model. The sensitivity to the SGS model and to the pressure-velocity coupling is shown to be small in the present study. The LES is compared with the available experimental data and ISO 5167-2. In general, the LES shows good agreement with the velocity from the experimental data. The profiles of the Reynolds stresses are similar, but an offset is observed in the diagonal stresses. The pressure loss and discharge coefficients are shown to be in very good agreement with the predictions of ISO 5167-2. Therefore, the wall-resolved LES is shown to be highly accurate in simulating the flow across a square-edged orifice.
\end{abstract}

\section{Keywords}

CFD, wall-resolved LES, orifice plate, discharge coefficient, pressure drop coefficient.

\section{Introduction}

Pressure differential devices remain the most common tools used to measure flow rates in industry. They work on the principle that variations in the flow pattern alter the pressure and velocity profiles. Pressure differential devices include diaphragms, nozzles, and Venturi tubes. They are favored since an international standard exists for regulating their use: ISO 5167. By respecting these standards, the flow rate measurements can be guaranteed within a quantifiable and commercially acceptable level of uncertainty.

The orifice flow meter is a commonly used instrument for flow measurements in pipes. Given the simplicity and reliability of the tool, it is used across many industries, as it is the case at EDF, for single phase flow measurements. A key variable affecting the measurement is the straight pipe length upstream and downstream of the orifice plate. The standards take this constraint into account by indicating a minimum straight pipe length upstream and downstream of the orifice. This constraint is not always met in practice due to other factors including plant layout, piping 
arrangement and equipment positioning. Computational Fluid Dynamics (CFD) can play a major role in the calculation of discharge coefficients outside the scope of the ISO standards, as it would be very expensive, if not impossible, to perform an experiment for every configuration. The involved Reynolds numbers (Re) might be of the order of $10^{5}$ to $10^{6}$, or even greater. It would then be unaffordable to perform parametric studies at such Reynolds numbers using fine techniques such as Large Eddy Simulation (LES). The only reasonable approach is the one based on Reynolds Averaged Navier-Stokes (RANS) equations.

The present study is the first step of a whole program which aims at using the RANS approach to compute different configurations. However, a RANS model sufficiently robust for this application must be selected first. For this purpose, a very fine LES is performed at $\mathrm{Re}=25000$ on a simple configuration and validated against the ISO standard and available experimental data. This will provide significantly more data of high quality to choose the RANS approach for the industrial computations. The present validation of LES will also provide confidence on its use if needed, at a Reynolds number of the order of $10^{4}$, for another configuration for which no experimental data are available.

The literature shows that wall-modelled Reynolds Averaged Navier-Stokes (RANS) simulations can reasonably predict the discharge coefficient (see section 3 for the definition of this coefficient), albeit with a high dependency on discretization errors and on the turbulence model. Erdal and Andersson (1997), in a two-dimensional axisymmetric simulation of an orifice plate with a standard and modified $\mathrm{k}-\varepsilon$ model, show that the pressure drop across the orifice plate is highly dependent on the grid refinement around the orifice plate and the turbulence model used. Shah et al. (2012) model the orifice flow meter with a standard k- $\varepsilon$ model and show that the pressure recovery downstream of the orifice plate is not well predicted. More recently, Shaaban (2014) shows that the realizable $\mathrm{k}-\varepsilon$ turbulence is within $1.4 \%$ of the ISO estimate of the discharge coefficient. Although RANS simulations could reasonably predict the discharge coefficient in certain simulations, first and second order statistics downstream of the orifice plate diverge significantly from experimental data. Erdal and Andersson (1997) demonstrate that the standard $\mathrm{k}-\varepsilon$ turbulence model does not accurately capture the physics of the flow especially around the region where the fluid accelerates across the pipe.

A review of the literature has not found a simulation technique that can reasonably predict both the pressure drop and first and second order statistics downstream of the plate. Therefore, it is of interest to study the precision obtainable with a wall-resolved Large Eddy Simulation (LES) and compare the results to detailed experimental data.

\section{Test-case}

The present LES is based on the test case of Shan et al. (2013) who used a planar Particle Image Velocimetry (PIV) to measure the flow field downstream of a circular square-edged orifice plate in a round pipe.

The computational domain of the test case is shown in Fig. 1. The relevant parameters are given in Table 1, and the pipe wall is assumed to be smooth. A dimensional analysis leads to three nondimensional numbers: The hydraulic Reynolds number $\mathrm{Re}$ and two aspect ratios, $\beta$ the ratio between the orifice and the pipe inner diameters and $\alpha$ the ratio between the orifice plate thickness and the pipe inner diameter. The values of these three non-dimensional parameters are equal to 
25000, 0.62 and 0.11, respectively. Note that in some references such as Idel'cik (1996), the ratio between the bulk surfaces $\beta^{2}$ is used instead of $\beta$.

In all the profiles provided in the present paper, the distances in the stream-wise direction are downstream of the center of the orifice plate and the pipe centerline is at $r / R=1$, where $R$ is the radius of the main pipe. The velocity components and the Reynolds stresses are made non-dimensional using the maximum mean velocity $u_{\max } \cdot u_{\max }$ is equal to $2.36 \mathrm{~m} / \mathrm{s}$ and $1.85 \mathrm{~m} / \mathrm{s}$ in the computations and in the experiment, respectively.

\section{Table 1.}

Test-case main parameters.

\begin{tabular}{|l|l|}
\hline Density $\rho\left[\mathrm{kg} / \mathrm{m}^{3}\right]$ & 996.65 \\
\hline Dynamic viscosity $\mu[\mathrm{kg} / \mathrm{m} / \mathrm{s}]$ & $8.5410^{-4}$ \\
\hline Main pipe inner diameter $D[\mathrm{~m}]$ & 0.046 \\
\hline Orifice diameter $d[\mathrm{~m}]$ & 0.0285 \\
\hline Orifice plate thickness $[\mathrm{m}]$ & 0.005 \\
\hline Bulk velocity in the main pipe $u_{0}[\mathrm{~m} / \mathrm{s}]$ & 0.466 \\
\hline
\end{tabular}

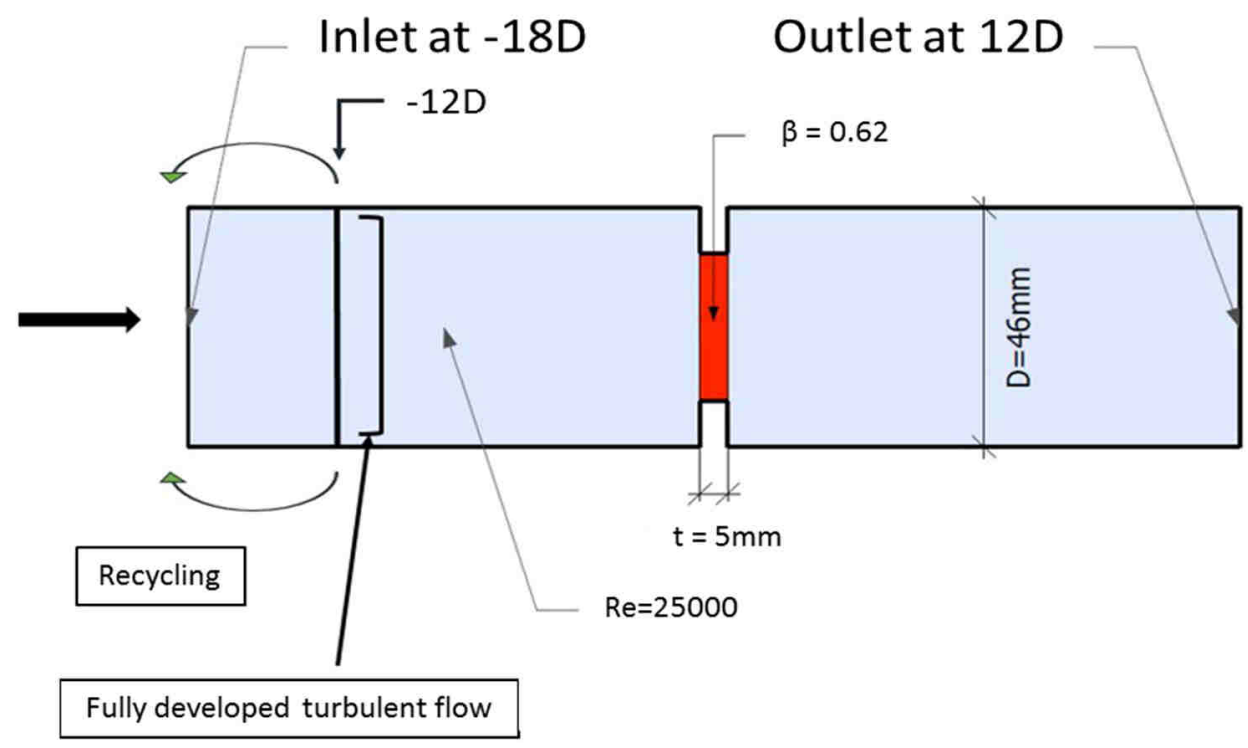

Fig. 1. Computational domain and boundary conditions.

\section{Discharge and Pressure Loss Coefficients}

The discharge coefficient $C_{D}$ is defined by (1):

$C_{D}=\frac{1}{\varepsilon} u_{0} \sqrt{1-\beta^{4}} \sqrt{\frac{\rho}{2 \Delta P_{D}}}$

where $\varepsilon$ is the coefficient of compressibility and $\Delta P_{D}$ the mean pressure drop between taps located $1 D$ upstream of the orifice and $0.5 D$ downstream (both measured from the upstream face of the 
contraction as required in ISO 5167-2, 2013). The relation that gives the bulk velocity and thus the flow rate is then given by (2):

$u_{0}=\frac{C_{D}}{\sqrt{1-\beta^{4}}} \varepsilon \sqrt{\frac{2 \Delta P_{D}}{\rho}}$

The coefficient of compressibility $\varepsilon$ is equal to 1 as the velocity of the fluid tackled in the present article leads to Mach numbers significantly below 0.3 .

The discharge coefficient and its uncertainty can be calculated using the Reader-Harris/Gallagher equation (see ISO 5167-2, 2013). The coefficient is a function of the geometry, the Reynolds number, the placement of the pressure taps and upstream and downstream flow features (such as bends, tees or valves). ISO TR12767 (1998) covers some deviations from the scope of ISO 51671 (2013) and ISO 5167-2 (2013).

The pressure loss coefficient $\xi$ is given by (3):

$\xi=\frac{2 \Delta P}{\rho u_{0}^{2}}$

where $\Delta P$ is the mean pressure drop between taps located $2 D$ upstream of the orifice and $6 D$ downstream. The stream-wise variation in the wall pressure is deemed to be linear at these locations.

The pressure loss coefficient $\xi$ can be estimated using the discharge coefficient in accordance with ISO 5167-2 (2013) from (4):

$\xi_{I S O}=\left(\frac{\sqrt{1-\beta^{4}\left(1-C_{D, I S O}^{2}\right)}}{C_{D, I S O} \beta^{2}}-1\right)^{2}$

The ISO 5167-2 (2013) estimates of the discharge and pressure drop coefficients are $0.628 \pm$ 0.005 and $8.71 \pm 0.07$, respectively. Note that the value obtained for the pressure drop coefficient using Idel'cik (1996) correlations is equal to 8.61 for the present configuration.

\section{Numerical approach}

\subsection{Large Eddy Simulation with Code_Saturne}

Code_Saturne is a highly customizable open source (www.code-saturne.org) CFD package developed by EDF (see Archambeau et al., 2004). It is based on a co-located finite volume discretization and deals with unstructured meshes with polyhedral cells of any type. It can deal with incompressible and compressible flows. Here, the flow is incompressible and the Velocity and pressure coupling is ensured via the SIMPLEC algorithm. The pressure Poisson equation is solved using an algebraic multigrid. The solver is parallelized (see Fournier et al., 2011) using MPI and OpenMP. Further information can be found in Archambeau et al. (2004). The LES capabilities of Code_Saturne have been validated on various academic and industrial cases including decaying isotropic turbulence, channel flows, side-by-side cylinders, tube bundles and gusts over a plate; see Benhamadouche (2006), Afgan et al. (2011) and Afgan et al. (2013) for example. 
The temporal discretization for the LES is second order Crank-Nicolson in time with linearized convection. The spatial discretization is a pure second order central difference scheme. By default, there are no external sweeps on the pressure-velocity coupling. The effect of the external sweeps is shown to be negligible in the present case (see subsection 5.2).

The sub-grid scale models used are the Dynamic Smagorinsky (Germano et al., 1991 and Lilly, 1992), as a base case, the standard Smagorinsky model (Smagorinsky, 1963) and no SGS model to test sensitivities. In the dynamic model, negative values of the Smagorinsky constant are not allowed and its maximum value is set de 0.065 . In the standard Smagorinsky model, the constant is set to 0.065 and a Van Driest damping function is used. Further details about the implementation of LES in Code_Saturne can be found in Benhamadouche (2006).

\subsection{Computational domain, boundary conditions and mesh details}

The inlet is located $18 D$ upstream of the orifice and the outlet is located $12 D$ downstream of the orifice (see Fig. 1). In all the profiles provided in this paper, the distances in the stream-wise direction are downstream of the center of the orifice plate and the pipe centerline is at $r / R=1$, where $R$ is the radius of the main pipe.

The inlet profile is simulated through a recycling method (see Lamballais, 2014) along $6 D$ (as shown in Fig. 1). This is to be distinguished from a precursor computation. At the first time step, artificial eddies are created using the synthetic eddy method (see Jarrin et al., 2006). Then, the flow 6D downstream of the inlet at a given time step is reused as an input for the next time step, creating a fully developed turbulent flow. The outlet uses a standard condition based on an imposed pressure and zero Neumann boundary conditions for the other variables. As the mesh is fine enough at the wall, no-slip boundary conditions are utilized.

The computational mesh is created using ICEM CFD v14.0. It is structured in the stream-wise direction, and locally refined in the stream-wise direction near the orifice. It also must be noted that the mesh is perfectly aligned in the stream-wise direction. This is important for the recycling method to generate the fully developed turbulent flow. Moreover, the mesh is conformal throughout the domain. This is also crucial, as non-conformal interfaces are known to be unsuitable in LES since they may introduce significant numerical errors with the present numerical approach. The non-dimensional wall distance $y^{+}$is kept below 1 across almost the whole domain, ensuring an accurate wall resolved LES. The refinement in the two other directions is also below the requirements for a wall resolved LES and this is imposed by the criterion applied in the regions far from the wall (see below the statements about the estimated Taylor micro-scale).

The cell size far from the wall is determined using precursor RANS results to ensure that the solution is resolved beyond an estimate of the Taylor micro-scale, which is taken equal to $\sqrt{15 v k / \varepsilon}$ (see Pope, 2000), where $k$ is the turbulent kinetic energy, $v$ the kinematic viscosity and $\varepsilon$ the turbulent dissipation rate. This roughly gives a maximum non-dimensional azimuthal length of 12 , a maximum non-dimensional radial length of 10, and a maximum non-dimensional stream-wise length of 40 (the values are based on the friction velocity upstream of the orifice). The final mesh consists of approximately 53 million cells. Fig. 2 shows the type of the mesh which has been used perpendicularly to the stream-wise direction. Fig. 3 represents the non-dimensional distance to the wall obtained a posteriori around the contraction. Locally, $y^{+}$can be higher than 1 (up to 1.5) but the influence is expected to be minimal. 


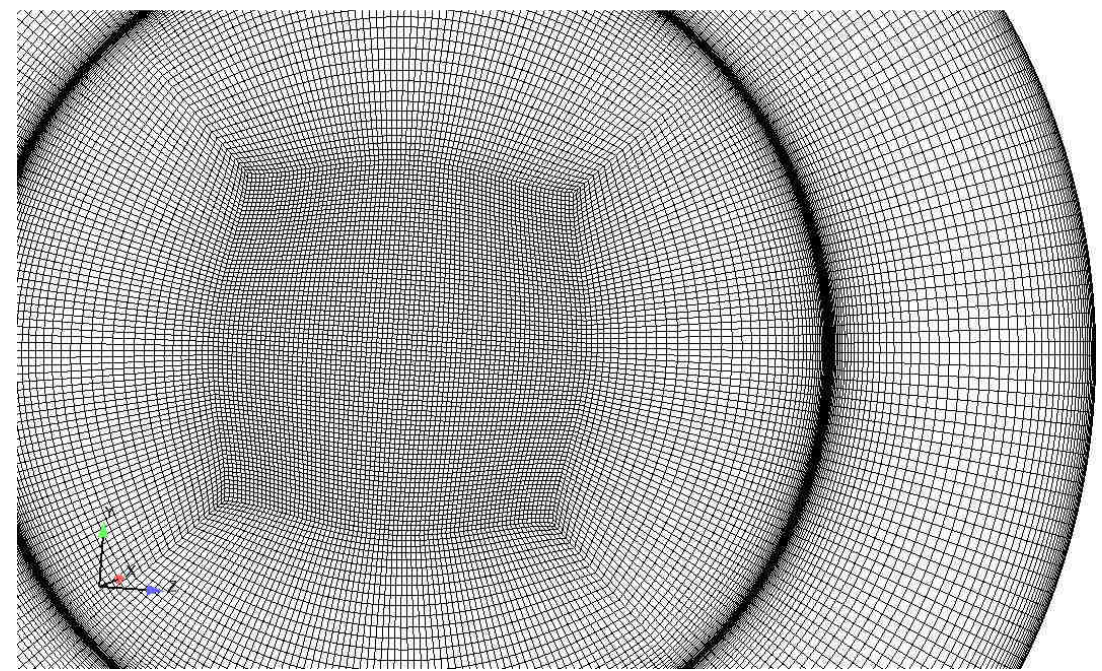

Fig. 2. Mesh view in a plane orthogonal to the stream-wise direction.

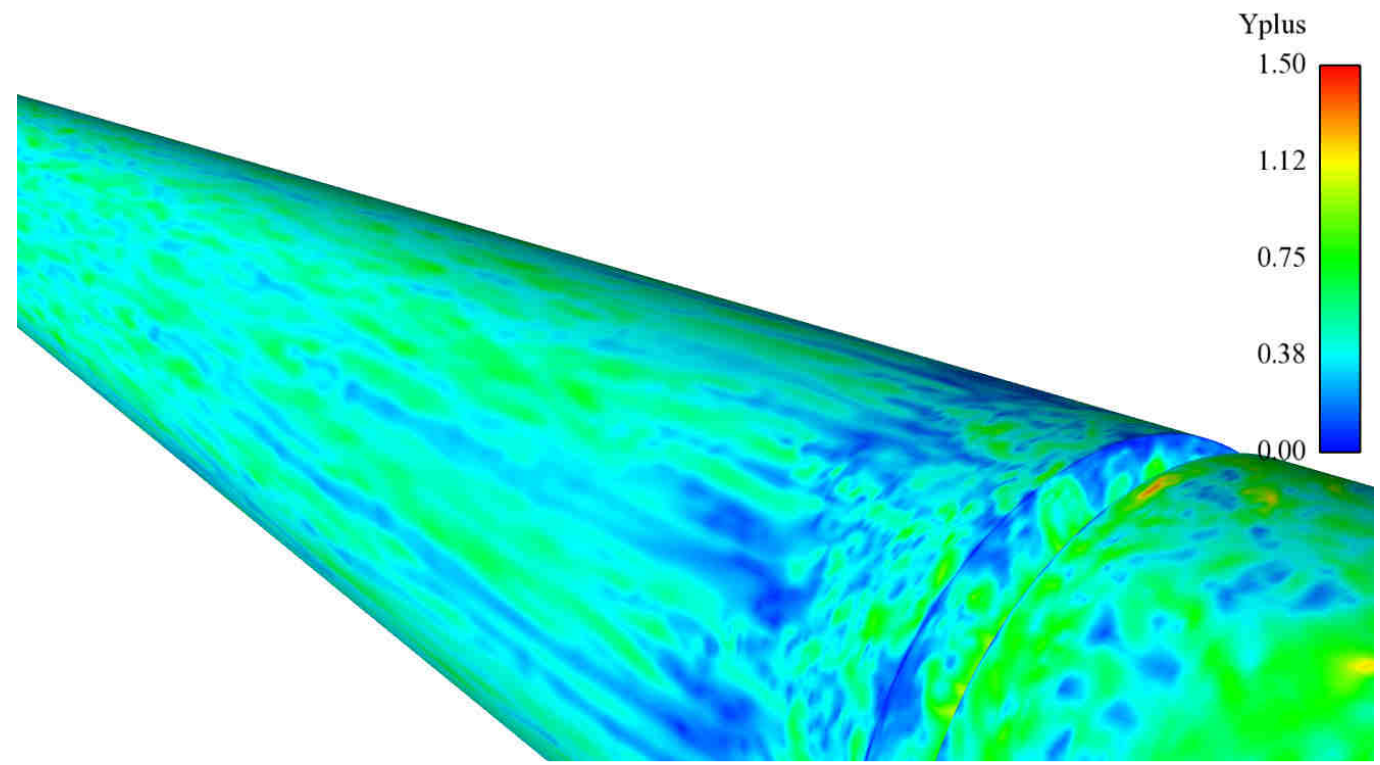

Fig. 3. Non-dimensional wall distance $y^{+}$of the first cell center (dynamic Smagorinsky model).

\subsection{Temporal statistic and axisymmetry of temporal averages}

The time step is constant, and it is initially chosen to ensure a CFL less than 1 throughout the solution domain. However, it is later increased (such that there are localized CFL values greater than one. Its initial and final values are equal to $0.510^{-5} \mathrm{~s}$ and $10^{-5} \mathrm{~s}$, respectively. As the time scheme is second-order implicit, these localized CFL values greater than one are deemed acceptable since they do not cause any observed numerical instabilities. 
The statistics start after 3 flow-passes in the computational domain downstream of the face used for recycling the inlet (length equal to 24D). This corresponds to 12 flow-passes in the recycled pipe (length equal to 6D). An instantaneous azimuthal velocity field in the recycled pipe is shown in Fig. 4 before starting collecting the statistics. The structures are obviously characteristic of a fully developed turbulent flow in a pipe. The non-dimensional wall distance $y^{+}$of the first cell shown in Fig. 3 exhibits the characteristic low speed streaks expected in the near-wall region (see Pope, 2000). An instantaneous stream-wise velocity field in the mid-plane near the orifice is also given in Fig. 5 to show that even after 1 flow-pass across the computational domain, the flow already looks fully developed. The first and second order statistics are collected in time during approximately 8 flow-passes across the computational domain downstream the face used for recycling (length equal to $24 \mathrm{D}$ ), corresponding to $1.2 \times 10^{6}$ iterations. The computations have been run on EDF Blue Gene/Q supercomputer using 4096 cores. The computational cost per timestep was around $2.2 \mathrm{~s}$.

The axisymmetry of the mean velocity gradient and the Reynolds stress tensor has been verified $a$ posteriori, at least close to the orifice plate and it the near wall region for the mean pressure. Fig. 6 shows the turbulent kinetic energy and the mean wall pressure coefficient $\left(C_{p}=p /\left(\frac{1}{2} \rho u_{0}^{2}\right)\right)$ profiles averaged in the azimuthal direction compared to local ones (not averaged in the azimuthal direction) after 6 flow-passes. The difference between the local profile and averaged profile is very small, and this is true for first and second order statistics. This is actually a very good indicator of the statistical convergence, at least in the considered regions.
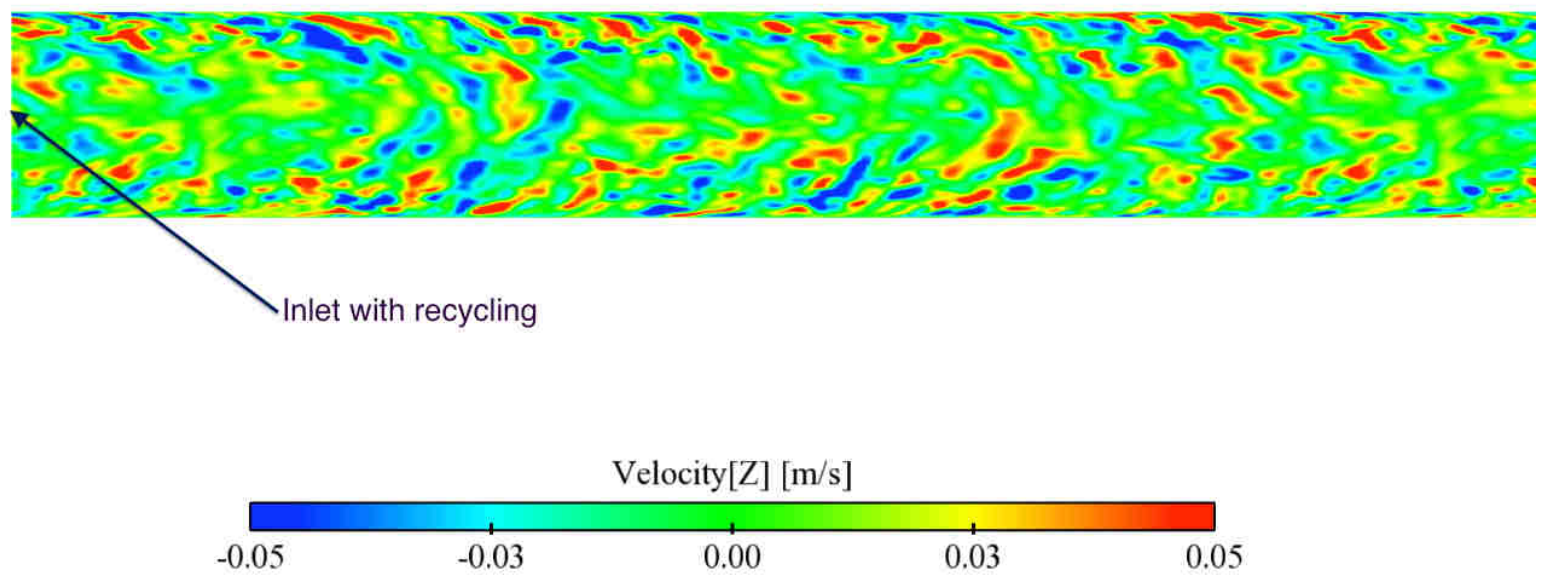

Fig. 4. Azimuthal (out of plane) instantaneous velocity field in the recycled pipe after approximately 5 flow-passes along 6D (dynamic Smagorinsky model). 

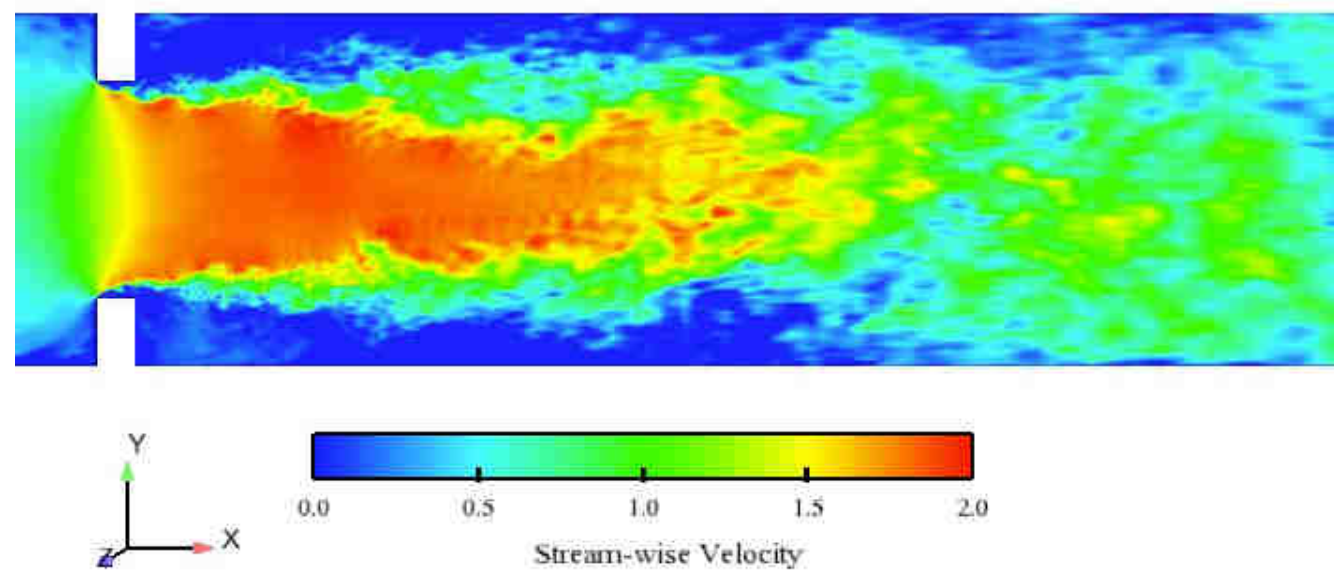

Fig. 5. Instantaneous stream-wise velocity after approximately 1 flow-pass across the entire domain (dynamic Smagorinsky model).
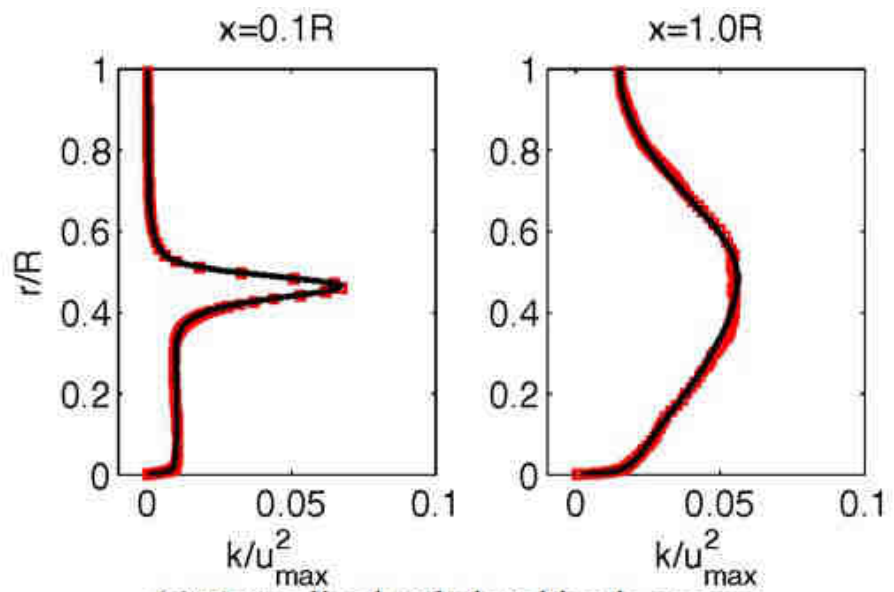

(a) Normalized turbulent kinetic energy.

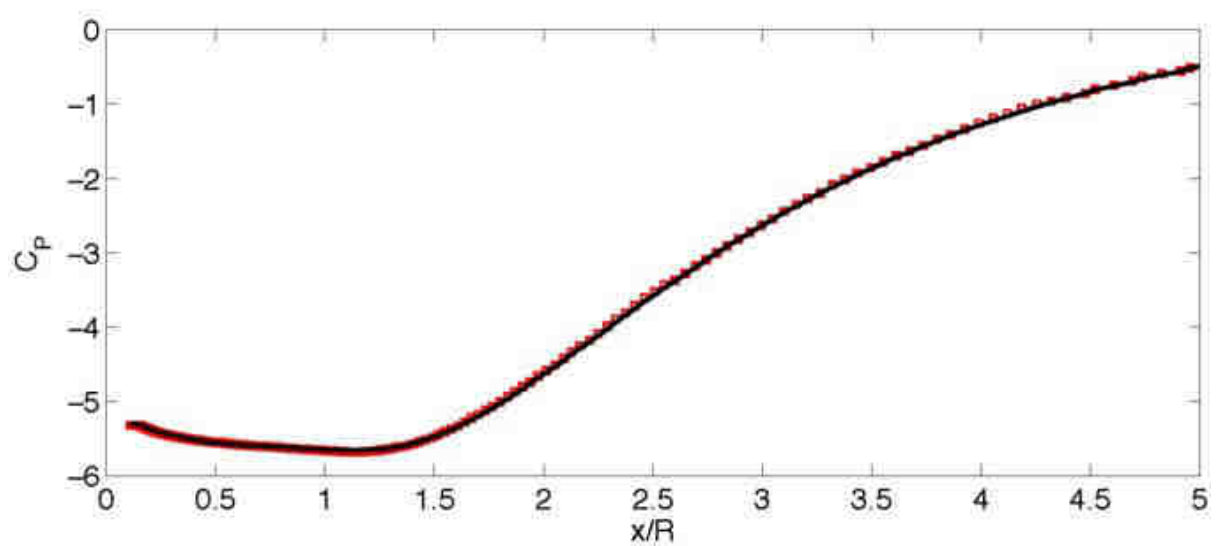

(b) Wall pressure coefficient

Fig. 6. Comparison between azimuthally and non-azimuthally averaged data (dynamic Smagorinsky model), $\square$ time average, - azimuthal and time average, $u_{\max }($ LES $)=2.36 \mathrm{~m} / \mathrm{s}$. 


\section{Sensitivity Studies}

Sensitivity studies of the LES are first performed evaluating the effect of the sub-grid scale model. Then, the effect of the number of external sweeps on the pressure-velocity coupling is examined. The sensitivity study is performed using time-averaged values.

\subsection{Sensitivity to the sub-grid scale model}

The most advanced sub-grid scale model used in the present study is the dynamic Smagorinsky one. Nonetheless, the standard Smagorinsky model and a simulation without a sub-grid scale model are compared. The profiles show no significant differences between the three different SGS models, as it can be seen in Fig. 7. Only the Reynolds stresses exhibit little differences (this is the reason for not showing the mean velocity component in Fig. 7). One can notice here that the dynamic Smagorinsky results are closer to the ones obtained by switching off the SGS model that to the ones obtained with a standard Smagorinsky. The very small differences between the three sub-grid scale models indicate that the modelling error is sufficiently small and that the mesh is fine enough to correctly predict first and second order statistics (one could call it Quasi-DNS).

Besides the profiles, the pressure loss coefficient and the three recirculation reattachment point distances are given in Table 2. Figs. 8 and 9 show the three reciculations in the present case and the definitions of $\mathrm{L}_{1}, \mathrm{~L}_{2}$ and $\mathrm{L}_{3}$. The recirculation reattachment points are determined at the point at which the wall shear stress changes direction, and are given in non-dimensional distances from the downstream face of the orifice plate (the radius $\mathrm{R}$ of the main pipe is use to make the distances non-dimensional). The overall pressure loss is not very sensitive to the SGS model. Its variations stay within the uncertainty given by the ISO. The primary reattachment length is more dependent on the sub-grid scales model than the pressure loss coefficients, what was not clearly visible using the mean velocity component. The most significant difference arises between the dynamic Smagorinsky model and the constant Smagorinsky model, while the LES without a SGS model is in between.

However, the close resemblance between all three models demonstrates that the LES is well resolved beyond the estimated Taylor micro-scale and wall-resolved, as the influence of the SGS model is low.

\subsection{Sensitivity to pressure-velocity coupling}

The number of sweeps (outer iterations) for the pressure-velocity coupling is increased with the dynamic Smagorinsky model. The standard cases only iterate once about the SIMPLEC pressure velocity coupling. In this test, the number of sweeps is increased to three, and it is averaged for approximately 4 flow-passes across the domain.

It is found that the differences between the two simulations are negligible in the present case (thus figures are not provided). For example, the relative difference between the predicted pressure loss coefficients is approximately $0.5 \%$, and the velocity and turbulent kinetic energy profiles do not change visually. It can thus be concluded that one sweep about the pressure-velocity coupling is sufficient in the present case. 

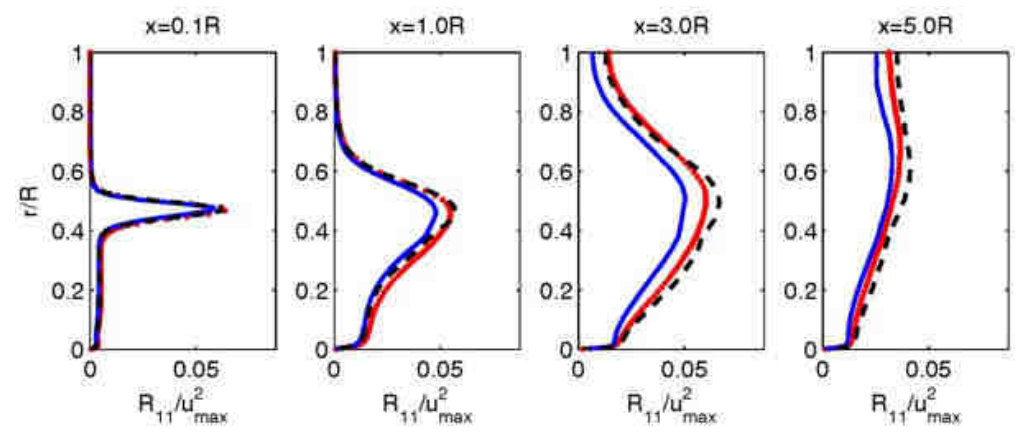

(a) Normalized $R_{11}=\overline{u_{x}^{\prime} u_{x}^{\prime}} / u_{\max }^{2}$
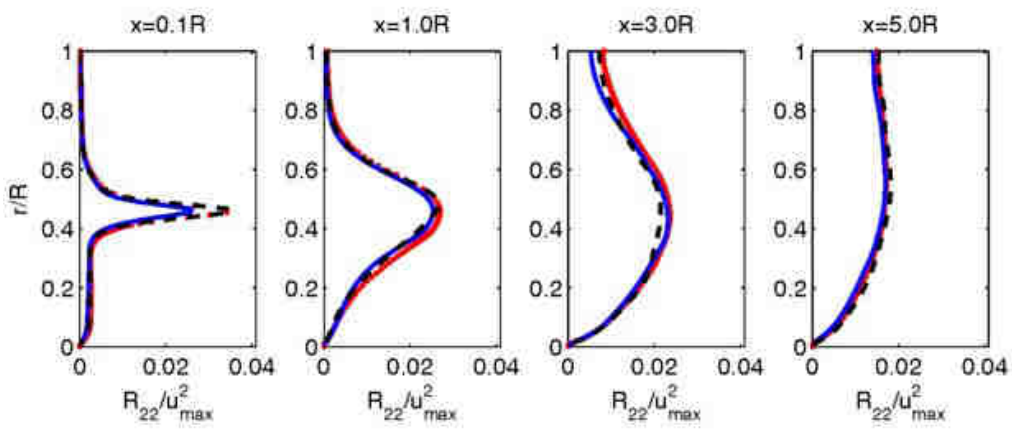

(b) Normalized $R_{22}=\overline{u_{r}^{\prime} u_{r}^{\prime}} / u_{\max }^{2}$
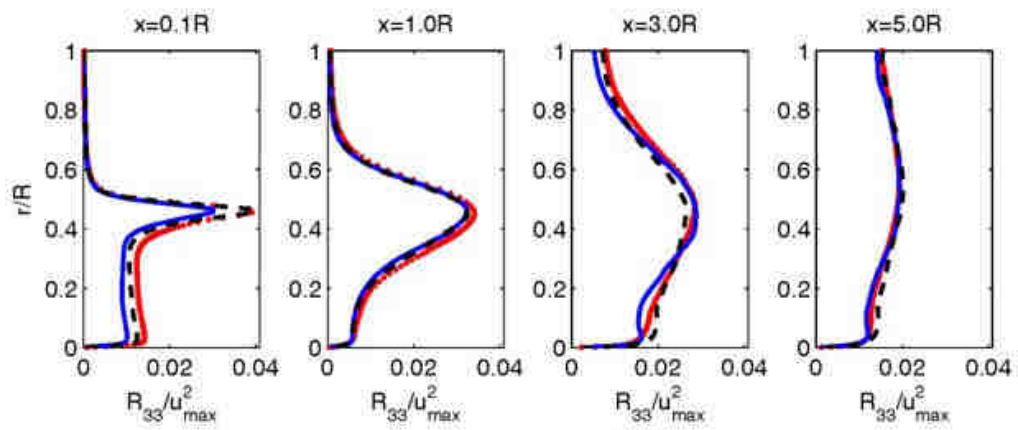

(c) Normalized $R_{33}=\overline{u_{\theta}^{\prime} u_{\theta}^{\prime}} / u_{\max }^{2}$
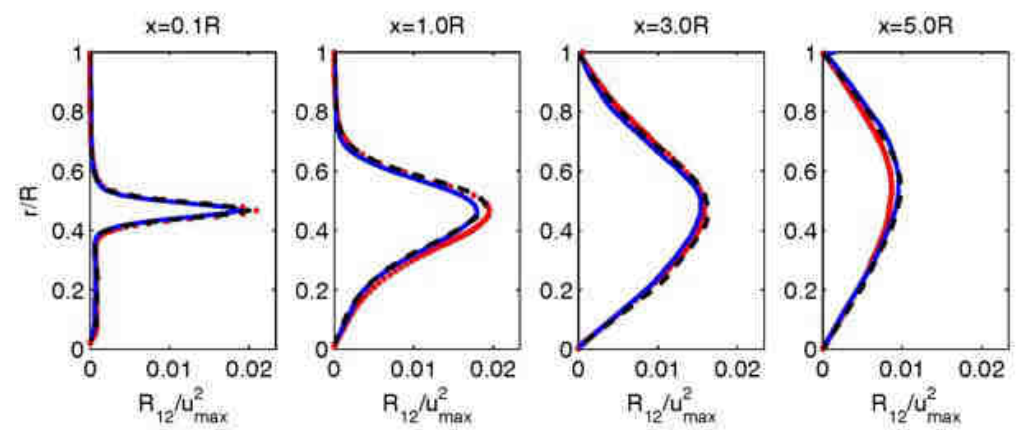

(d) Normalized $R_{12}=\overline{u_{x}^{\prime} u_{r}^{\prime}} / u_{\text {max }}^{2}$

Fig. 7. Comparison between different SGS models. $\square$ dynamic Smagorinsky, - standard Smagorinsky, - - - no SGS, $\mathrm{u}_{\max }(\mathrm{LES})=2.36 \mathrm{~m} / \mathrm{s}$. 


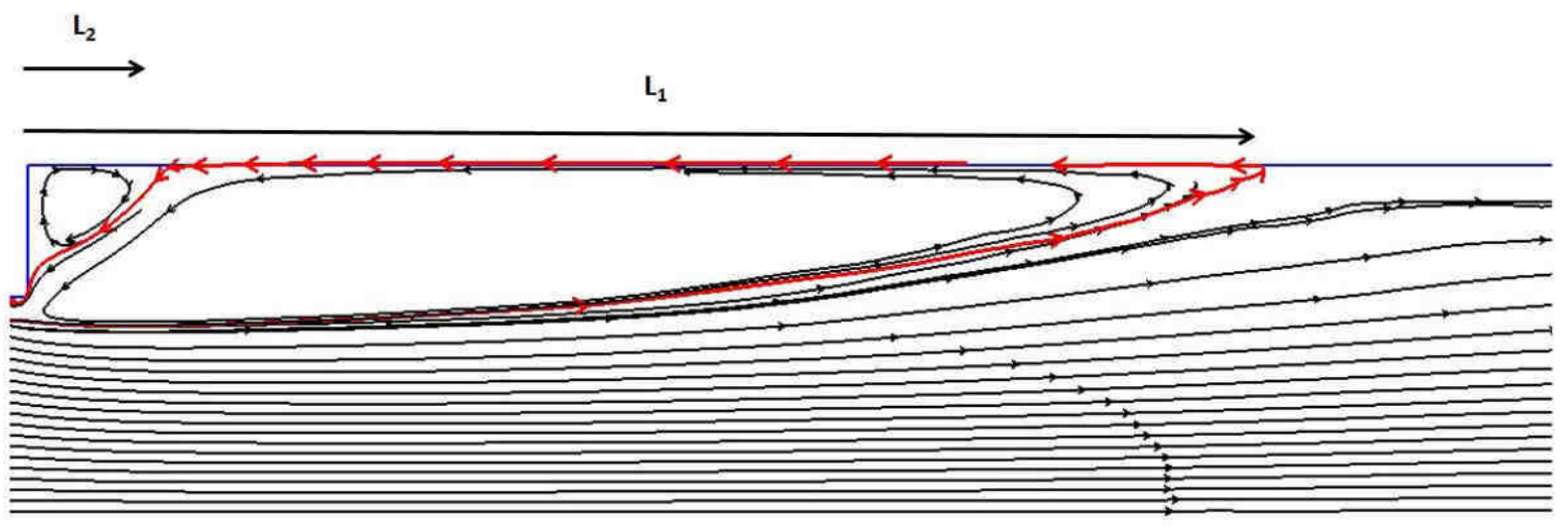

Fig. 8. Visualization of the primary $\left(\mathrm{L}_{1}\right)$ and secondary $\left(\mathrm{L}_{2}\right)$ recirculations

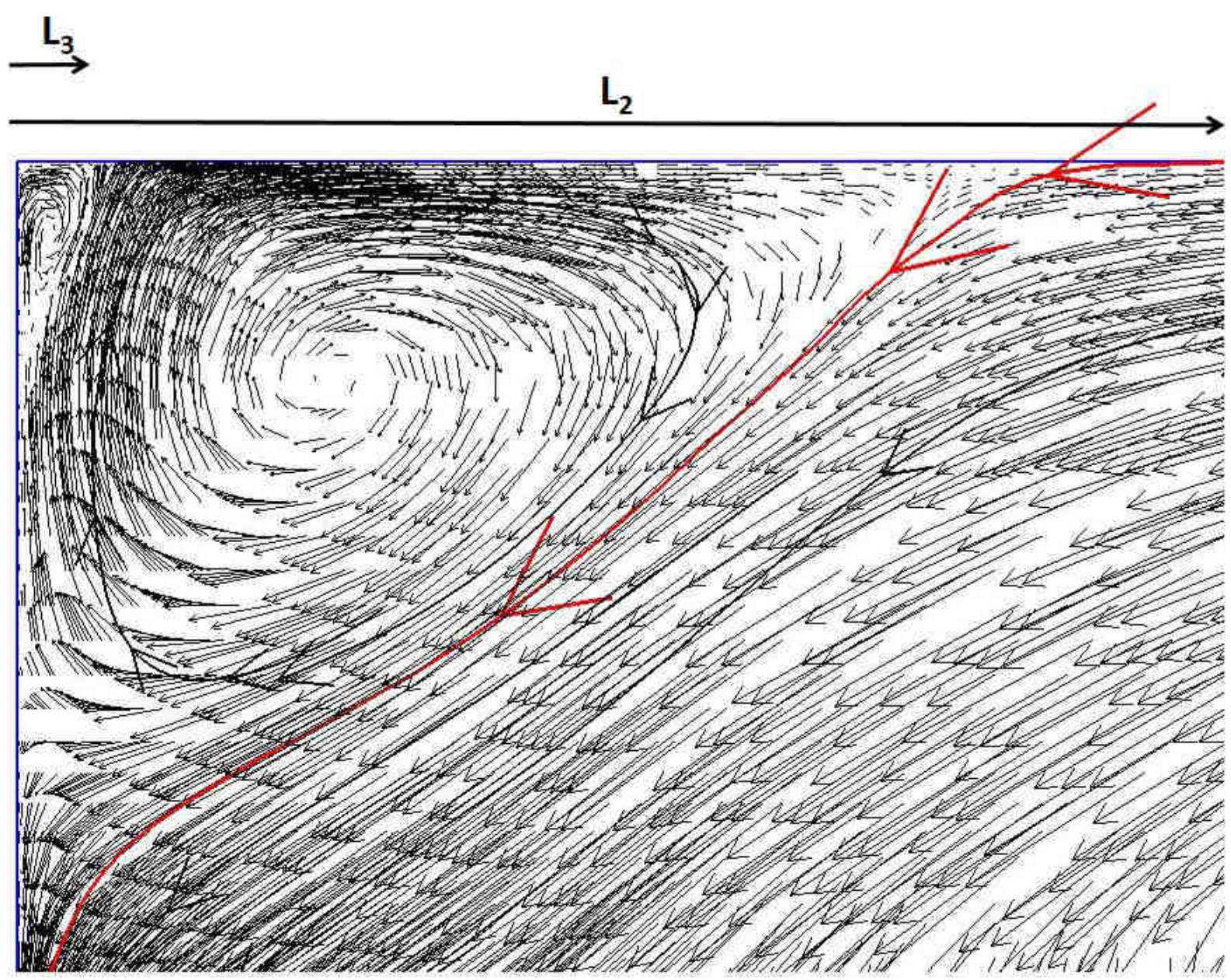

Fig. 9. Visualization of the secondary $\left(\mathrm{L}_{2}\right)$ and tertiary $\left(\mathrm{L}_{3}\right)$ recirculations 
Table 2.

Recirculation lengths and pressure loss coefficient for the three LES simulations.

\begin{tabular}{|l|c|c|c|c|}
\cline { 2 - 5 } \multicolumn{1}{c|}{} & $\begin{array}{l}\text { Dynamic } \\
\text { Smag. }\end{array}$ & $\begin{array}{l}\text { Standard } \\
\text { Smag. }\end{array}$ & $\begin{array}{l}\text { No SGS } \\
\text { Model }\end{array}$ & ISO \\
\hline Pressure loss coefficient & 8.64 & 8.79 & 8.71 & $8.71 \pm 0.07$ \\
\hline Primary reattachment $\left[\boldsymbol{L}_{\mathbf{1}} / \boldsymbol{R}\right]$ & 3.92 & 4.25 & 4.11 & - \\
\hline Secondary reattachment $\left[\boldsymbol{L}_{\mathbf{2}} / \boldsymbol{R}\right]$ & 0.42 & 0.37 & 0.40 & - \\
\hline Tertiary reattachment $\left[\boldsymbol{L}_{\mathbf{3}} / \boldsymbol{R}\right]$ & 0.025 & 0.020 & 0.023 & - \\
\hline
\end{tabular}

\section{Comparisons with experimental data}

LES with the dynamic Smagorinsky model is compared with PIV data from Shan et al. (2013). Fig. 10 gives the centerline stream-wise velocity downstream of the orifice, normalized by the maximum average velocity. The tail end of the centerline velocity differs slightly, but the results of the LES and PIV overall seem to be in very good agreement. This is an indicator for the very good predictions of the discharge and pressure drop coefficients.

Moreover, the shapes of both the LES and PIV stream-wise and radial velocity profiles provide a close match, as seen in Figs. 11(a) and 11(b). The results differ in the regions of high gradients of velocity. With regards to the results in the regions of high gradients, the resolution of the PIV is larger than the grid size of the LES; thus probably smoothing the results. For example, near $r / R=$ 0.6, as seen in Fig. 11(b), the LES predicts larger variations than the PIV for the radial velocity. At this point, the LES radial mesh length is $0.0018 \mathrm{D}$, while the PIV pixel size is $0.014 \mathrm{D}$, almost 8 times larger. Lastly, the normalized mean Reynolds stresses $R_{11}, R_{22}$ and $R_{12}$ corresponding to $\overline{u_{x}^{\prime} u_{x}^{\prime}}, \overline{u_{r}^{\prime} u_{r}^{\prime}}$ and $\overline{u_{x}^{\prime} u_{r}^{\prime}}$, respectively, can be compared in Figs. 11(c)-11(e). It is noticed that the experimental and LES profiles of $R_{12}$ are reasonably similar with the exception of areas with large gradients as shown. On the other hand, the normalized profiles of $R_{11}$ and $R_{22}$ show large differences even outside regions with large gradients. The values of experimental data are suspicious at the wall as they do not vanish. . This is not surprising as the PIV methodology is known to lose accuracy in the near wall region. The difference between the experimental and simulated $R_{11}$ and $R_{22}$ profiles is almost constant, and the qualitative behavior is the same. It would be very hazardous to speculate on the origin of the differences in the core region far from the area showing very high gradients.

The PIV predicts a reattachment length of the primary recirculation zone $x=3.64 R$ downstream of the orifice using the forward flow probability (FFP) method (see Shan et al., 2013). From the LES, the reattachment length is estimated at $x=3.92 R$ downstream of the orifice. However, the difference between the data is due to the method with which the reattachment length is calculated. The LES estimate determines the reattachment length as the point at which the wall shear stress changes direction. Since the near wall region is resolved, the velocity distribution is linear in the radial direction (normal to the wall); therefore $\tau_{w a l l}=\mu \frac{u^{(1)}}{r^{(1)}}$ where $u^{(1)}$ is the velocity at the centre of the first cell from the wall, and $r^{(1)}$ is the distance of that cell center to the wall. This is 
analogous to the mean stream-wise velocity zero-crossing estimate, where the reattachment zone is the point at which the velocity changes sign.

The PIV estimate of the reattachment point uses a FFP at $r=0.056 R$ away from the wall which corresponds to twice the resolution of the technique adopted (see Shan et al., 2013). The FFP method calculates a probability density function of the stream-wise velocity being positive. The reattachment length is then given by the point at which the probability of the velocity being positive is exactly $50 \%$. Using the experimental data, it is possible to estimate the recirculation zone using a mean stream-wise velocity zero-crossing method. The first point located at $r=0.028 R$ away from the wall is used to estimate the velocity. This method predicts a primary reattachment length $L_{1}=3.62 R$ downstream of the orifice, and the secondary reattachment length is found equal to $L_{2}=0.27 R$. A similar approach for the LES results in a primary reattachment length $L_{1}=3.60 R$ and a secondary reattachment length $L_{2}=0.34 R$. Therefore, it is clear that the primary and secondary reattachment points calculated with the same methodology using PIV data and the LES are very similar.

The discharge coefficient is calculated by taking pressure taps $1 D$ upstream of the orifice and $0.5 D$ downstream (both measured from the upstream face as required in ISO 5167-2, 2013). The dynamic Smagorinsky model gives a discharge coefficient $C_{D}=0.632$. The ISO 5167-2 estimate for the discharge coefficient is $0.628 \pm 0.005$. The results are very close.

The pressure loss across is measured between 2D upstream and 6D downstream, where the streamwise variation in the wall pressure is deemed to be linear. Using the dynamic Smagorinsky model, the pressure loss coefficient is $\xi=8.64$. Similarly, the estimates from ISO 5167-2 and from Idel'cik (1996) correlations are equal to $8.71 \pm 0.07$ and 8.61, respectively. The results are again very close.

The results between the ISO standards and the LES are, for both the discharge coefficient and the pressure loss coefficient, in very close agreement - within the margin of error of the ISO estimate - which serves as further validation of the LES results.

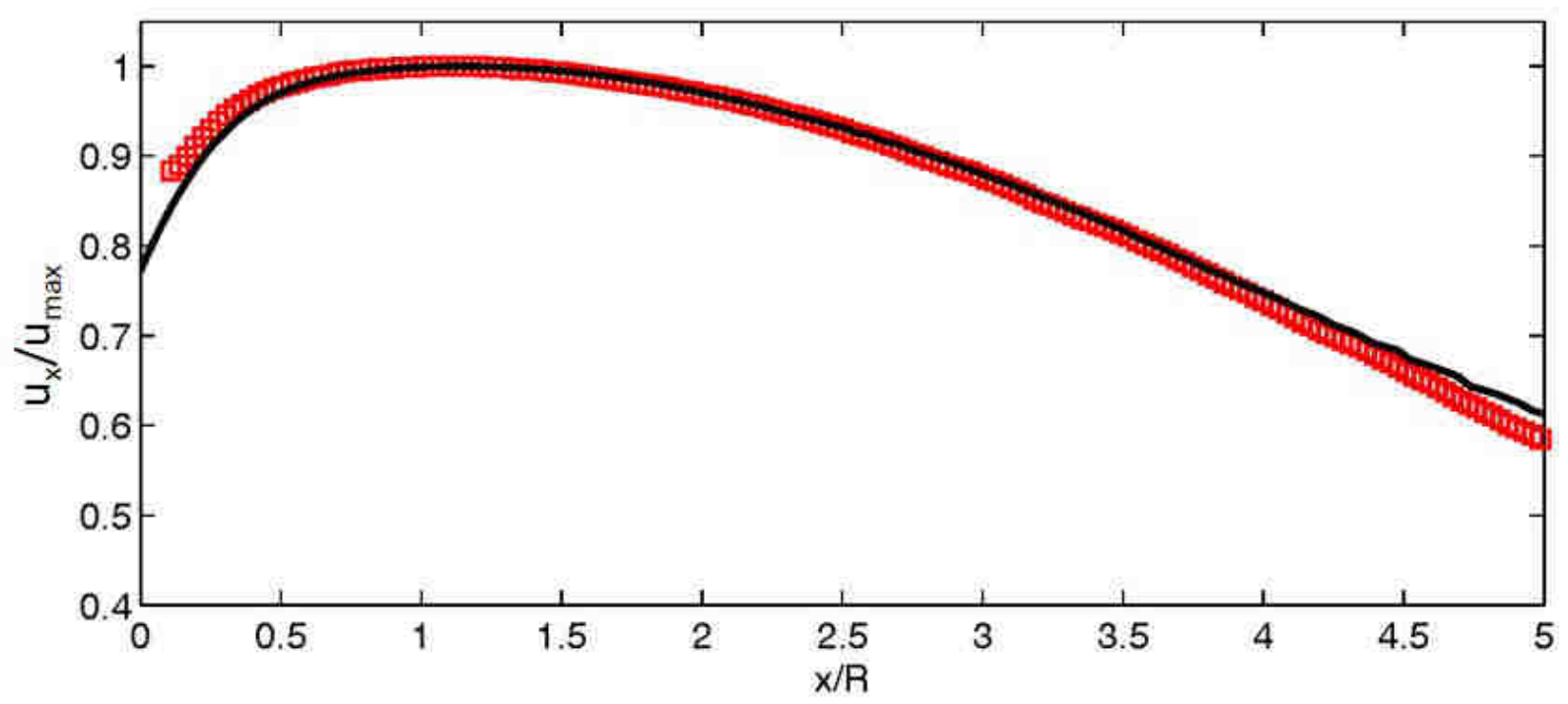

Fig. 10. Stream-wise centerline velocity profile downstream of the orifice. $\square$ experimental data, LES, $\mathrm{u}_{\max }(\exp )=1.85 \mathrm{~m} / \mathrm{s}, \mathrm{u}_{\max }(\mathrm{LES})=2.36 \mathrm{~m} / \mathrm{s}$. 


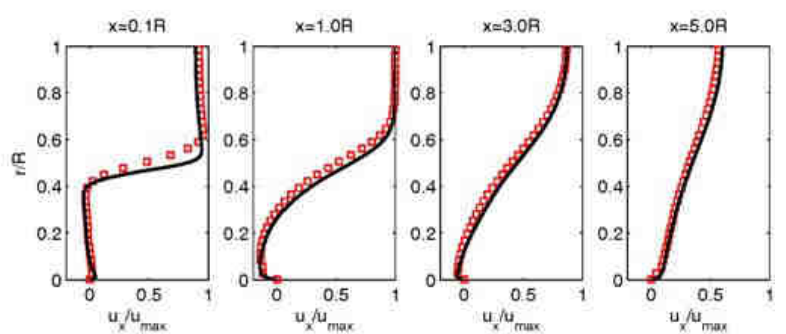

(a) Normalized stream-wise velocity $u_{x} / u_{\max }$

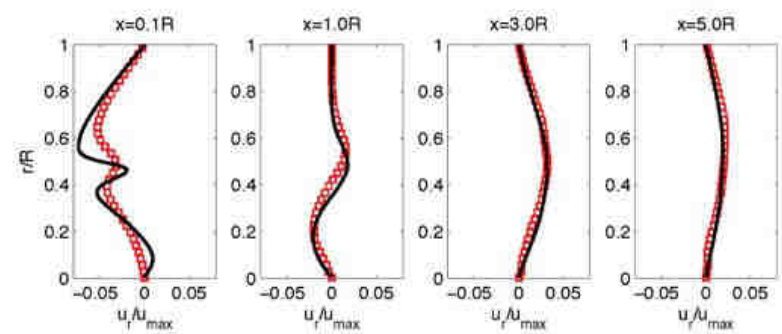

(b) Normalized radial velocity $u_{r} / u_{\max }$

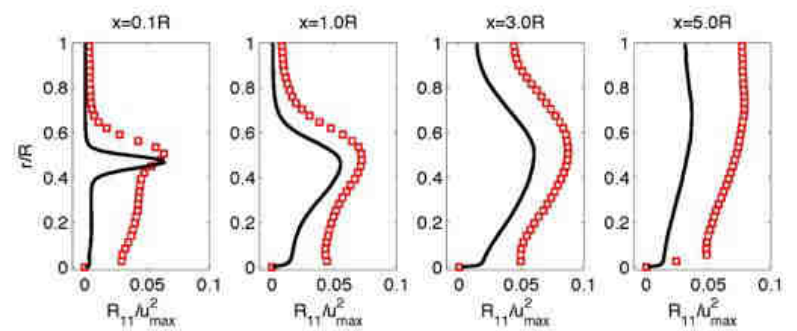

(c) Normalized $R_{11}=\overline{u_{x}^{\prime} u_{x}^{\prime}} / u_{\max }^{2}$

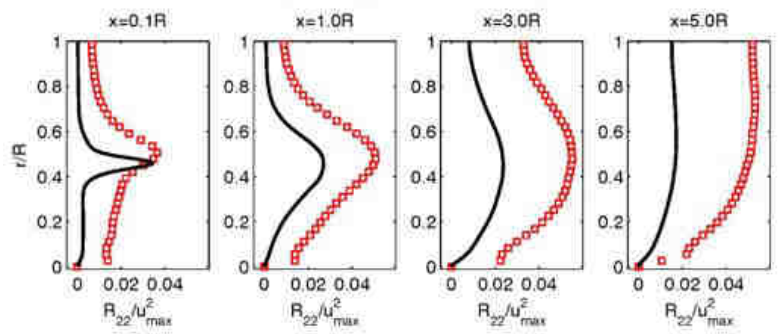

(d) Normalized $R_{22}=\overline{u_{r}^{\prime} u_{r}^{\prime}} / u_{\text {max }}^{2}$

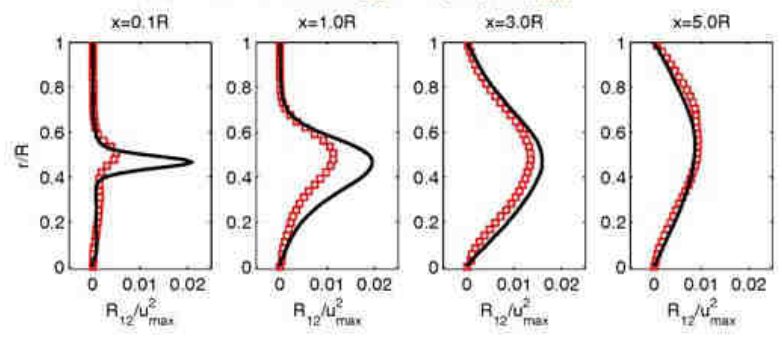

(e) Normalized $R_{12}=\overline{u_{x}^{\prime} u_{r}^{\prime}} / u_{\max }^{2}$

Fig. 11. Comparison between LES and PIV profiles. $\square$ experimental data, - LES, $\mathrm{u}_{\max }(\exp )=1.85$ $\mathrm{m} / \mathrm{s}, \mathrm{u}_{\max }(\mathrm{LES})=2.36 \mathrm{~m} / \mathrm{s}$. 


\section{Conclusions}

The present study demonstrates that a very fine wall-resolved LES with a dynamic Smagorinsky SGS can accurately and precisely simulate a single phase flow through a square-edged orifice flow meter at Re of the order $10^{4}$. A sensitivity study shows that the effect of the SGS model and pressure-velocity coupling is small.

The LES shows excellent agreement with the velocity from the experimental data. There is also good agreement observed between the respective $R_{12}$ profiles. However, the $R_{11}$ and $R_{22}$ profiles show an offset but a good qualitative agreement. Moreover, the recirculation lengths are well predicted by the LES. Lastly, the pressure loss and discharge coefficients are also shown to be in very good agreement with the predictions of ISO 5167-2 (2003).

Therefore, the wall-resolved LES is shown to be accurate in simulating the flow across a squareedged orifice. The results from this simulation can be used to validate other simulation techniques such as RANS approaches or provide sufficient confidence to compute other configurations at moderate Reynolds numbers. Moreover, this study shows that the LES is a viable technique in predicting recirculation lengths, discharge coefficient, pressure loss coefficient, mean velocities and Reynolds stresses in an orifice flow meter. This has important repercussions for the assessment of heat and mass transfer characteristics of industrial orifice flow meters. This result also shows that the discharge coefficients and pressure loss coefficients should be predictable using the LES technique in non-standard configurations.

\section{Acknowledgements}

The authors would like to acknowledge Dr. Feng Shan for kindly sharing the experimental results for the present test case.

\section{References}

Afgan, I., Benhamadouche, S., Han, X., Sagaut, P., Laurence, D., 2013. Flow over a flat plate with uniform inlet and incident coherent gusts. Journal of Fluid Mechanics 720, 457-485.

Afgan, I., Kahil, Y., Benhamadouche, S., Sagaut, P., 2011. Large eddy simulation of the flow around single and two side-by-side cylinders at subcritical Reynolds numbers. Physics of Fluids 23(7), 075101.

F. Archambeau, F., Mechitoua, N., Sakiz, M., 2004. Code_Saturne: A finite volume method for the computation of turbulent incompressible flows: Industrial applications. International Journal on Finite Volumes, Electronical edition: http://averoes.math.univ-paris13.fr/html ISSN 1634 (0655).

Benhamadouche, S., 2006. Large-Eddy Simulation with the unstructured collocated arrangement. $\mathrm{PhD}$ thesis, The University of Manchester.

Erdal, A., Andersson, H.I., 1997. Numerical aspects of flow computation through orifices. Flow Measurement and Instrumentation 8(1), 27-37.

Fournier, Y., Bonelle, J., Moulinec, C., Shang, Z., Sunderland, A.G., Uribe, J.C., 2011. Optimizing Code_Saturne computations on Petascale systems. Computers \& Fluids 3(7), 1760-1765. 
Germano, M., Piomelli, U., Moin, P., Cabot, W., 1991. A dynamic subgrid-scale eddy viscosity model. Physics of Fluids 3(7), 1760-1765.

Idel'cik, I.E., 1996. Handbook of Hydraulic Resistance. Third Edition, Begell House Inc.

ISO 5167-1, 2003. Measurement of fluid flow by means of pressure differential devices inserted in circular cross-section conduits running full -- Part 1.

ISO 5167-2, 2003. Measurement of fluid flow by means of pressure differential devices inserted in circular cross-section conduits running full -- Part 2.

ISO TR 12767, 1998. Measurement of Fluid Flow by Means of Pressure Differential DevicesGuidelines to the Effect of Departure from the Specifications and Operating Conditions given in ISO, 5167.

Jarrin, N., Benhamadouche, S., Laurence, D., Prosser, R., 2006. A synthetic-eddy-method for generating inflow conditions for large-eddy simulations. International Journal of Heat and Fluid Flow 27(4), 585-593.

Lamballais, E., 2014. Direct numerical simulation of a turbulent flow in a rotating channel with a sudden expansion. Journal of Fluid Mechanics 745, 92-131.

Lilly, D., 1992. A proposed modification of the Germano subgrid-scale closure method. Physics of Fluids 4(3), 633-635.

Pope, S.B., 2000. Turbulent flows, Cambridge university press.

Shaaban, S., 2014. Optimization of orifice meter's energy consumption. Chemical Engineering Research and Design 92(6), 1005-1015.

Shah, M.S., Joshi, J.B., Kalsi, A.S., Prasad, C.S.R., Shukla, D.S., 2012. Analysis of flow through an orifice meter: CFD simulation. Chemical Engineering Science 71, 300-309.

Shan, F., Fujishiro, A., Tsuneyoshi, T., Tsuji, Y., 2013. Particle image velocimetry measurements of flow field behind a circular square-edged orifice in a round pipe. Experiments in Fluids 54(6), $1-18$.

Smagoinsky, J., 1963. General circulation experiments with the primitive equations. I. The basic equations. Monthly Weather Review 91(3), 99-164. 